\title{
Requerimiento de hemostasia endoscópica en pacientes de una unidad de cuidado intensivo con hemorragia de vías digestivas altas
}

\section{Endoscopic hemostasis in intensive care unit patients with upper digestive tract bleeding}

\author{
Daniel Medina Torres ${ }^{1}$, William Otero Regino ${ }^{2^{*}}$, Elder Otero Ramos ${ }^{3}$
}

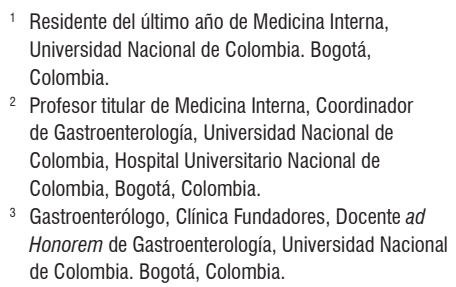

2 Profesor titular de Medicina Interna, Coordinador de Gastroenterología, Universidad Nacional de Colombia, Hospital Universitario Nacional de Colombia, Bogotá, Colombia.

3 Gastroenterólogo, Clínica Fundadores, Docente ad Honorem de Gastroenterología, Universidad Nacional de Colombia. Bogotá, Colombia.

*Correspondencia: William Otero, waoteror@gmail.com

Fecha recibido: $28 / 01 / 19$ Fecha aceptado: 27/03/19

\begin{abstract}
Resumen
Introducción: los pacientes hospitalizados en la unidad de cuidados intensivos (UCI) tienen riesgo de hemorragia digestiva alta (HVDA). La endoscopia digestiva alta (EVDA) es el examen de elección en esos pacientes y es diagnóstica y terapéutica. Muchas lesiones identificadas endoscópicamente no requieren tratamiento endoscópico. En Colombia no hay estudios sobre la prevalencia de las diferentes lesiones sangrantes digestivas altas en pacientes de la UCl, ni sobre la utilización de EVDA terapéutica en esos pacientes. Materiales y métodos: estudio de corte transversal realizado en la Clínica Fundadores de Bogotá, Colombia, entre enero del 2003 a febrero del 2017. Se incluyeron pacientes adultos de la unidad de cuidado intensivo con EVDA indicada por HVDA. Resultados: en el análisis final se incluyeron 156 EVDA. Los hallazgos fueron los siguientes: gastritis crónica 76,62\% (118), esofagitis erosiva (grado A-grado D) 57,79\% (89), gastritis erosiva $47,4 \%$ (73), duodenitis erosiva 21,43\% (33), úlcera gástrica 18,18\% (28), varices esofágicas $11,69 \%$ (18), úlcera duodenal $11,04 \%$ (17) y desgarro de Mallory-Weiss 4,55\% (8). Solo el $15 \%$ de los pacientes requirió manejo endoscópico, incluidos los que tenían várices esofágicas. Conclusión: en el presente estudio, el 15\% de los pacientes con HVDA requirió tratamiento endoscópico. Se deben realizar trabajos prospectivos que permitan establecer factores de riesgo que puedan predecir la necesidad de EVDA terapéutica en pacientes con HVDA. Quien no tenga esos predictores se debe tratar empíricamente con IBP y evitar gastos innecesarios en EVDA diagnósticas.
\end{abstract}

Palabras clave

Cuidados intensivos, hemorragia, endoscopia.

\begin{abstract}
Introduction: Patients hospitalized in an intensive care unit (ICU) are at risk of upper gastrointestinal bleeding. Esophagogastroduodenoscopy (EGD) is the test of choice for these patients. EGD is diagnostic and therapeutic. Many endoscopically identified lesions do not require endoscopic treatment. In Colombia there are no studies on the prevalence of different upper gastrointestinal bleeding lesions in ICU patients, nor on the use of therapeutic EGD in these patients. Materials and methods: This is a cross-sectional study conducted at the Clínica Fundadores in Bogotá Colombia between January 2003 and February 2017. Adult ICU patients who underwent EGD due to upper gastrointestinal bleeding were included. Results: In the final analysis, 156 patients who underwent EGD were included. Of these, $76.62 \%$ (118) had chronic gastritis, $57.79 \%$ (89) had erosive esophagitis grades A to D, 47.4\% (73) had erosive gastritis, $21.43 \%$ (33) had erosive duodenitis, $18.18 \%$ (28) had gastric ulcer, $11.69 \%$ (18) had esophageal varices, $11.04 \%$ (17) had duodenal ulcers, and $4.55 \%$ (8) Mallory Weiss tears. Only $15 \%$ of patients, including those with esophageal varices, required endoscopic management. Conclusions: In this study, $15 \%$ of patients with upper gastrointestinal bleeding required endoscopic treatment. Prospective work should be done to establish risk factors to predict the need for therapeutic EGD in patients with upper gastrointestinal bleeding. Patients do not have these predictors should be treated empirically with PPI to avoid unnecessary expenses of diagnostic EGDs.
\end{abstract}

\section{Keywords}

Intensive care, hemorrhage, endoscopy. 


\section{INTRODUCCIÓN}

Los pacientes hospitalizados en la unidad de cuidados intensivos (UCI) tienen mayor riesgo de hemorragia digestiva alta (HVDA), especialmente por úlceras de estrés $(1,2)$. Su aparición se asocia con resultados adversos, que incluyen aumento de la mortalidad ( 2 a 4 veces) y una mayor estancia en la UCI ( 4 a 8 días más) $(3,4)$. La incidencia del sangrado gastrointestinal en la UCI varía de $0,17 \%$ a $7,0 \%(3,5)$. La endoscopia de las vías digestivas altas (EVDA) es el examen de elección para los pacientes con HVDA, incluidos los de UCI, y es tanto diagnóstico (examen macroscópico de las lesiones, toma de biopsias) como terapéutico (hemostasia con diferentes modalidades) $(5,6)$. Su desempeño está bien demostrado en pacientes con HVDA (7-10). Sin embargo, frecuentemente también se realiza en pacientes admitidos a la UCI por otros motivos; en estos casos, las indicaciones son mucho más debatidas; además, el descubrimiento de una lesión de la mucosa puede no requerir ningún tratamiento endoscópico ni farmacológico (gastritis, esofagitis, entre otros) $(5,7,11,12)$.

Dentro de las causas de HVDA en pacientes de la UCI están las várices esofágicas o gástricas, esofagitis, úlceras y gastritis erosivas hemorrágicas relacionadas con el estrés (13). Se ha estimado que hasta el $90 \%$ de los pacientes críticos puede presentar daño de la mucosa gastroduodenal después de 3 días de hospitalización en UCI y ese daño puede progresar a úlceras y producir sangrado, así como también sangrado por gastritis erosiva (14). Sin embargo, el sangrado clínicamente importante ocurre en solo $2 \%$ a $3 \%$ y las úlceras por estrés se identifican endoscópicamente como la fuente del sangrado en menos del $50 \%$ de esos pacientes $(4,15,16)$. Estos datos han planteado la discusión sobre la necesidad real de la EVDA, teniendo en cuenta los costos y el impacto real que tendría su utilización indiscriminada en ese tipo de pacientes, más aún porque solo un pequeño porcentaje progresa hacia un sangrado gastrointestinal manifiesto y clínicamente importante (17).

Otras afecciones menos comunes responsables del sangrado alto son el síndrome de Mallory-Weiss y las lesiones vasculares (13). Los principales factores de riesgo identificados para VDA en UCI incluyen ventilación mecánica por más de 48 horas, coagulopatía activa, enfermedad hepática y renal $(5,18,19)$. Otros factores de riesgo son el choque, falla hepática y renal, sepsis, trauma múltiple, quemaduras de más del $35 \%$ de la superficie corporal, trasplante de órganos, trauma de cráneo o raquimedular, antecedente de enfermedad ulcerosa previa e hipoalbuminemia (20-24).

En nuestro medio no encontramos publicaciones sobre la prevalencia de las diferentes lesiones sangrantes del tracto digestivo superior en pacientes hospitalizados en la
UCI, como tampoco la frecuencia de hemostasia endoscópica en ellos. Teniendo en cuenta la escasa información disponible se decidió realizar el presente trabajo en la Unidad de Gastroenterología de la Clínica Fundadores de Bogotá, identificando los pacientes con HDA de la UCI a quienes se les hubiera realizada EVDA.

\section{MATERIALES Y MÉTODOS}

El presente es un estudio de corte transversal basado en los hallazgos de EVDA de pacientes de la UCI de la Clínica Fundadores, que requirieron el procedimiento por HVDA. Se incluyeron los pacientes adultos mayores de 18 años, atendidos durante el período comprendido entre el 1 de enero 2003 al 31 de diciembre del 2017.

\section{Criterios de inclusión}

Se incluyeron pacientes hospitalizados en UCI por enfermedades críticas, quienes presentaron HVDA después de 24 horas de hospitalización y se les realizó EVDA.

\section{Criterios de exclusión}

Pacientes de UCI a quienes se les hubiera realizado EVDA por indicación diferente a HVDA. También se excluyeron los pacientes con EVDA incompleta, los hospitalizados en la unidad de cuidados intermedios y mujeres embarazadas. No se incluyeron quienes estaban hospitalizados en UCI por HVDA severa.

La información fue obtenida de los informes de EVDA de la unidad de gastroenterología correspondiente a la UCI y la revisión de la respectiva historia clínica de los pacientes identificados. Las diferentes variables de cada paciente fueron registradas y consignadas en un instrumento de recolección de datos elaborado para la presente investigación. Debido a que solo se incluían pacientes con EVDA se desconoce el número de pacientes que existieron durante el período de estudio en UCI con HVDA a los que no se les realizó endoscopia.

\section{Objetivo general}

Determinar el requerimiento de hemostasia endoscópica en pacientes en la unidad de cuidado intensivo con hemorragia de vías digestivas altas.

\section{Objetivos específicos}

- Determinar la prevalencia de lesiones endoscópicas en pacientes con hemorragia digestiva alta hospitalizados en la unidad de cuidado intensivo. 
- Identificar las técnicas empleadas en el abordaje endoscópico y comparar la prevalencia de hallazgos endoscópicos según los grupos etarios.

\section{Análisis estadístico}

Las variables cualitativas se presentaron en forma de números absolutos y proporciones, específicamente se calculó la prevalencia de la siguiente forma: prevalencia $=$ (número de pacientes con el hallazgo endoscópico/total de la población recogida) $x 100$. La prevalencia se estratificó por los distintos grupos etarios. Finalmente, las variables cuantitativas se presentaron en forma de medida de resumen y dispersión según su distribución estadística, la cual fue evaluada mediante la prueba de Shapiro-Wilk. Se consideró una significancia estadística cuando el valor de $p$ era menor de 0,05.

\section{RESULTADOS}

Durante el período de estudio se practicaron 246 EVDA a paciente de la UCI. Se excluyeron 92 (69 por indicación diferente a sangrado de vías digestivas altas (18 para paso de sonda nasoentérica, 8 para gastrostomía endoscópica, 7 por neoplasia, 6 por dolor abdominal, 4 por anemia, 4 por dispepsia, 3 por cirrosis conocida, 3 por varices esofágicas, 2 por reflujo gastroesofágico, 2 por trastorno de la deglución, 2 por esofagitis, 2 por intoxicación exógena, 1 por obstrucción esofágica, 1 por obstrucción intestinal, 1 por fístula intestinal, 1 por fístula traqueoesofágica, 1 por sangrado por biopsia, 1 por mediastinitis, 1 por herida por proyectil de arma de fuego, 1 por esofagectomía y ascenso gástrico), 13 por indicación no registrada, 6 estudios incompletos, 3 por preparación inadecuada y 1 paciente que no permitió la realización del examen; finalmente se incluyeron 154 EVDA (Figura 1); hubo 99 hombres $(64,29 \%)$ y 55 mujeres $(35,71 \%)$, con una mediana de edad de 68 años, la mitad de ellos entre 59 y 76 años.

Los hallazgos en la EVDA a nivel del esófago, estómago y duodeno se muestran en las Figuras 2-4, respectivamente.

Algún tratamiento endoscópico de las diferentes lesiones se realizó en 24 pacientes $(15,58 \%)$ y no fue necesario en los restantes 130 pacientes $(84,4 \%)$ (Figura 5). Dentro de los diagnósticos registrados en las historias clínicas para hallazgo de varices esofágicas, la principal patología fue la cirrosis Child Pugh C con encefalopatía, siendo en su mayoría secundaria a diabetes mellitus y alcohol. Sin embargo, para el resto de los hallazgos se presenta información ambivalente debido al gran número de patologías y comorbilidades con las que cursan los pacientes críticos (sepsis, choque, falla cardíaca, insuficiencia renal, falla respiratoria, coagulopatía, entre otras).

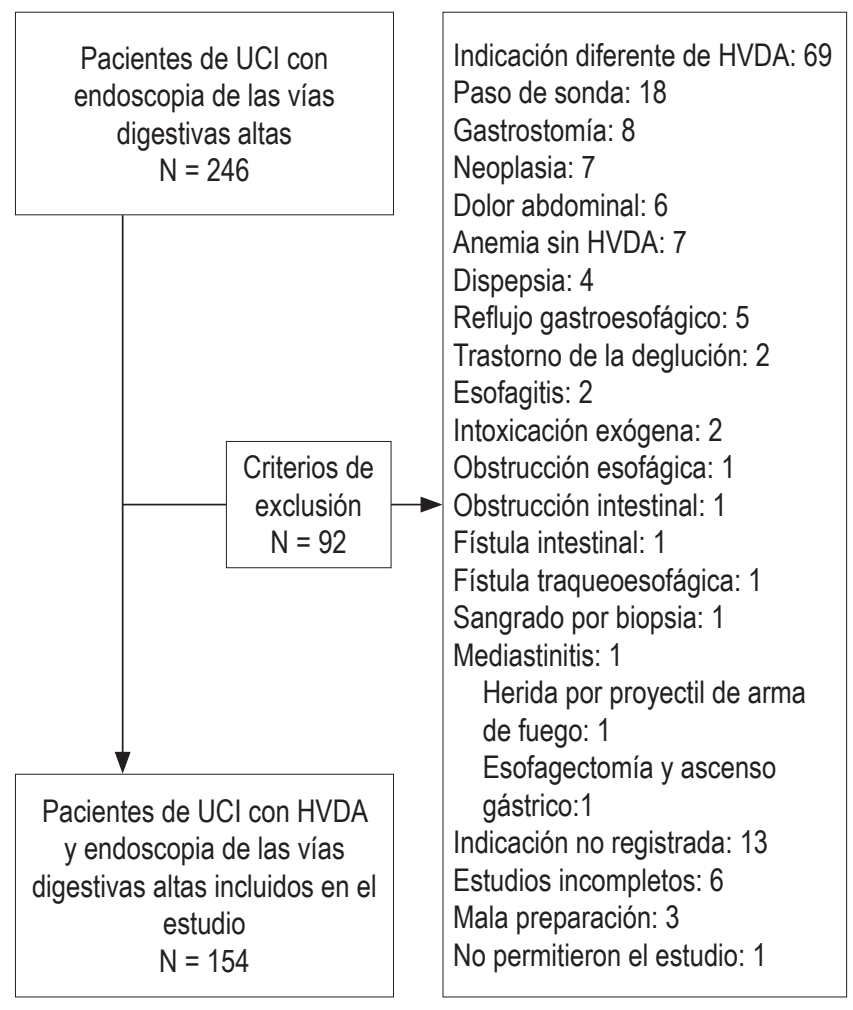

Figura 1. Diagrama de pacientes incluidos en el estudio.

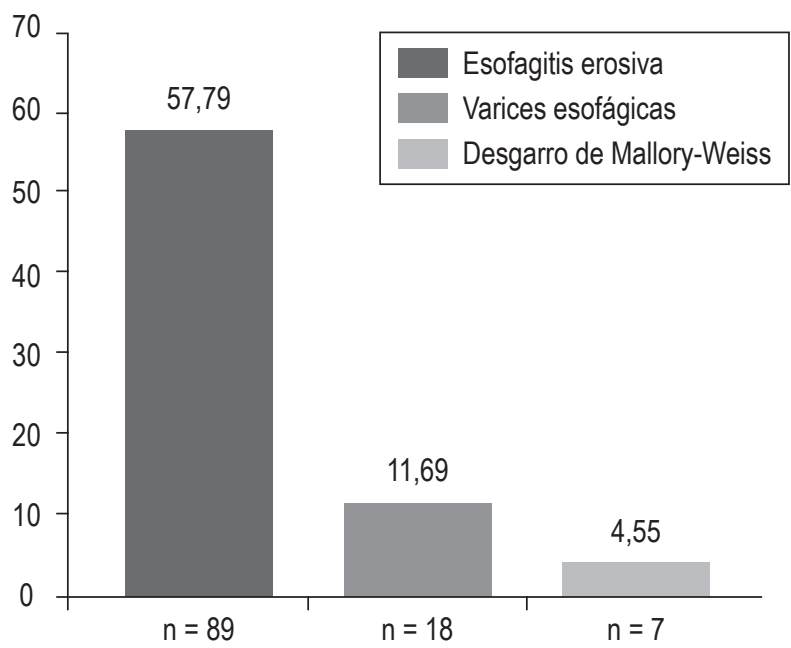

Figura 2. Hallazgos esofágicos en la EVDA.

Las diferentes modalidades endoscópicas terapéuticas utilizadas se muestran en la Tabla 1. En todos los pacientes se logró hemostasia de las lesiones sangrantes.

La prevalencia de las principales patologías responsables del ingreso a UCI se muestra en la Tabla 2. 


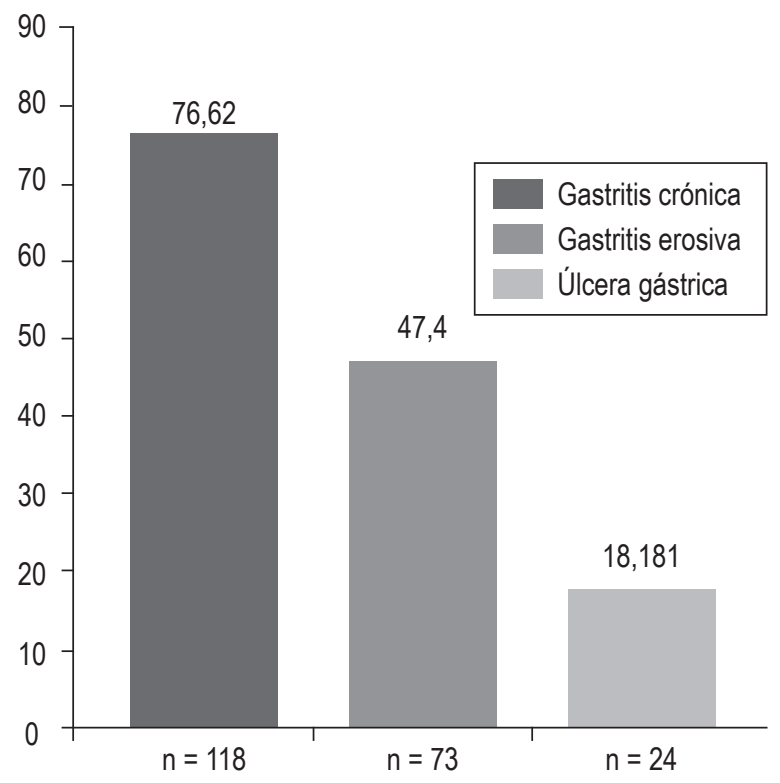

Figura 3. Prevalencia de hallazgos endoscópicos en el estómago.

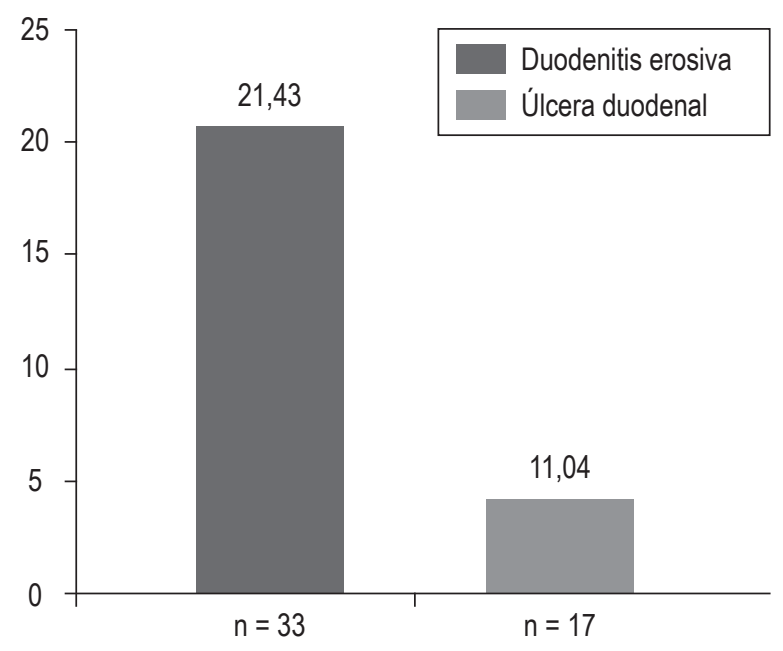

Figura 4. Hallazgos endoscópicos en el duodeno.

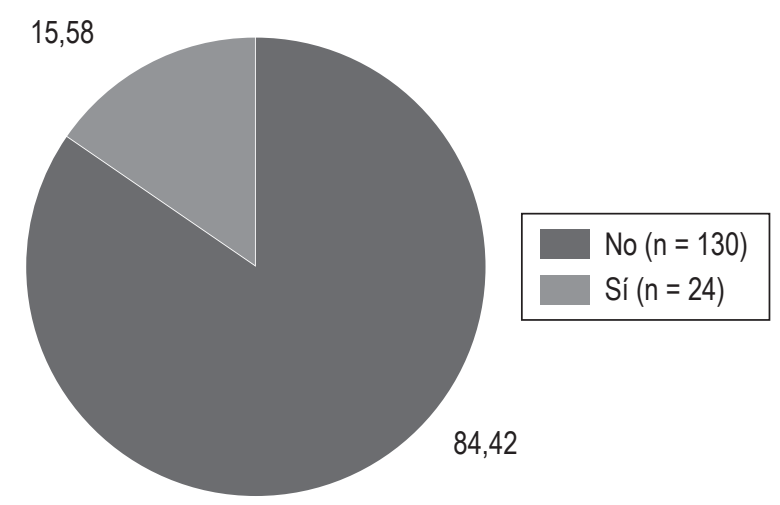

Figura 5. Tratamiento endoscópico.
Tabla 1. Modalidades terapéuticas en las lesiones sangrantes

\begin{tabular}{lcc}
\hline \multicolumn{1}{c}{ Lesiones } & \multicolumn{2}{c}{ Tratamiento endoscópico } \\
\cline { 2 - 3 } & $\begin{array}{c}\text { Terapia de } \\
\text { inyección con } \\
\text { adrenalina }\end{array}$ & $\begin{array}{c}\text { Ligadura } \\
\text { de várices } \\
\text { esofágicas }\end{array}$ \\
\hline $\begin{array}{l}\text { Úlceras gástricas: } 5,85 \% \\
\text { Varices esofágicas: } 4,55 \%\end{array}$ & $\mathrm{n}=9$ & $\mathrm{n}=7$ \\
$\begin{array}{l}\text { Úlceras duodenales: } 3,89 \% \\
\begin{array}{l}\text { Desgarro de Mallory-Weiss: } 1,29 \% \\
\text { Prevalencia del tratamiento } \\
\text { endoscópico: } 15,58 \%\end{array}\end{array}$ & $\mathrm{n}=6$ & \\
\hline
\end{tabular}

Tabla 2. Prevalencia de las patologías responsables de ingreso a UCI

\begin{tabular}{lcc}
\hline \multirow{1}{*}{ Patología } & \multicolumn{2}{c}{ Prevalencia } \\
\cline { 2 - 3 } & $\mathbf{N}$ & $\%$ \\
\hline Enfermedad pulmonar & 19 & 12,36 \\
Enfermedad cardiovascular & 38 & 24,69 \\
Sepsis & 31 & 20,17 \\
Cirugía mayor & 8 & 5,13 \\
Trauma & 8 & 5,13 \\
Enfermedad neurológica & 12 & 7,79 \\
Enfermedad hepática & 24 & 15,59 \\
Lesión renal aguda & 6 & 3,95 \\
Cetoacidosis diabética & 8 & 5,19 \\
\hline Total & 154 & 100 \\
\hline
\end{tabular}

La prevalencia de ventilación mecánica fue del 20\% (n = 30) y la profilaxis con inhibidores de bomba de protones (IBP) fue del 32\% ( $\mathrm{n}=49)$. La distribución de las lesiones por grupo etario se muestra en la Tabla 3.

\section{DISCUSIÓN}

En el presente estudio, el 15\% de los pacientes con HVDA hospitalizados en UCI requirió tratamiento endoscópico. La necesidad de EVDA terapéutica encontrada es similar a un estudio de la Universidad de Pensilvania en el que se estudiaron 66 pacientes de la UCI, y 15\% ameritó terapia endoscópica hemostática (25). Otro estudio realizado por Kim y colaboradores en la Universidad de Seúl en 66 pacientes encontró una prevalencia para manejo endoscópico del sangrado en un $19 \%$ (26).

La endoscopia de vías digestivas altas en pacientes críticos con hemorragia digestiva es frecuentemente solicitada tanto para fines diagnósticos como terapéuticos; sin embargo, distintas lesiones de la mucosa identificadas por este medio no requieren ningún tratamiento endoscópico, lo que aumenta los costos derivados de la atención del paciente y la probabilidad de complicaciones $(7,27)$. En general, estos pacientes mueren a causa de la gravedad de la 
Tabla 3. Prevalencia de hallazgos endoscópicos según grupo etario

\begin{tabular}{lcccccccc}
\hline \multirow{2}{*}{ Edad } & \multicolumn{7}{c}{ Prevalencia de hallazgos endoscópicos } \\
\cline { 2 - 9 } & $\begin{array}{c}\text { Gastritis } \\
\text { crónica }\end{array}$ & $\begin{array}{c}\text { Úlcera } \\
\text { gástrica }\end{array}$ & $\begin{array}{c}\text { Gastritis } \\
\text { erosiva }\end{array}$ & $\begin{array}{c}\text { Duodenitis } \\
\text { erosiva }\end{array}$ & $\begin{array}{c}\text { Úlcera } \\
\text { duodenal }\end{array}$ & $\begin{array}{c}\text { Esofagitis } \\
\text { erosiva }\end{array}$ & $\begin{array}{c}\text { Varices } \\
\text { esofágicas }\end{array}$ & $\begin{array}{c}\text { Desgarro de } \\
\text { Mallory-Weiss }\end{array}$ \\
\hline $20-30$ & $66,67 \%$ & $11,11 \%$ & $33,33 \%$ & $55,56 \%$ & $11,11 \%$ & $44,44 \%$ & $11,11 \%$ & $11,11 \%$ \\
$31-40$ & $100 \%$ & $0,0 \%$ & $66,67 \%$ & $0,0 \%$ & $33,33 \%$ & $33,33 \%$ & $0,0 \%$ & $0,0 \%$ \\
$41-50$ & $72,73 \%$ & $9,09 \%$ & $63,64 \%$ & $0,0 \%$ & $0,0 \%$ & $45,45 \%$ & $0,0 \%$ & $0,0 \%$ \\
$51-60$ & $80 \%$ & $16,0 \%$ & $44 \%$ & $20 \%$ & $12 \%$ & $64 \%$ & $20 \%$ & $4 \%$ \\
$61-70$ & $74,42 \%$ & $16,28 \%$ & $41,86 \%$ & $16,28 \%$ & $13,95 \%$ & $51,16 \%$ & $11,63 \%$ & $0,0 \%$ \\
$71-80$ & $79,07 \%$ & $25,58 \%$ & $53,49 \%$ & $23,26 \%$ & $9,3 \%$ & $65,12 \%$ & $6,98 \%$ & $11,63 \%$ \\
$81-90$ & $70,59 \%$ & $23,53 \%$ & $47,06 \%$ & $29,41 \%$ & $5,88 \%$ & $64,71 \%$ & $23,53 \%$ & $0,0 \%$ \\
$>90$ & $100 \%$ & $0,0 \%$ & $33,33 \%$ & $33,33 \%$ & $33,33 \%$ & $66,67 \%$ & $0,0 \%$ & $0,0 \%$ \\
\hline
\end{tabular}

condición médica subyacente o por disfunción multiorgánica más que por causa del sangrado (28).

Lee y colaboradores realizaron un estudio prospectivo en 105 pacientes de unidad de cuidado crítico con sangrado gastrointestinal y encontraron una prevalencia para enfermedad erosiva del 21,9\% (11). En nuestro estudio, la gastritis erosiva presentó una prevalencia del $47,4 \%(n=73)$, sin embargo, ninguna de estas lesiones requirió tratamiento endoscópico. Por lo anterior, la relevancia clínica de estas lesiones es controvertida, ya que solo un pequeño porcentaje progresa hacia un sangrado gastrointestinal manifiesto y clínicamente importante $(17,22)$.

A nivel esofágico, el hallazgo más frecuente fue la esofagitis erosiva, la cual la presentó más de la mitad de los pacientes (59\%), superior a lo publicado previamente $(25,29-31)$. Este hallazgo demuestra nuevamente que en la mayoría de las EVDA de pacientes de UCI con HVDA se encontrarán lesiones que no ameritan endoscopia terapéutica, por tanto, no habrá un impacto en el manejo de los pacientes (7). El desgarro de Mallory-Weiss (DMW) se presentó en 8 pacientes (4,55\%). En ninguno de estos pacientes se necesitó terapia endoscópica. El DMW está relacionado con el aumento súbito de la presión intragástrica o intraabdominal, que se trasmite a la unión esofagogástrica (32). Los factores de riesgo incluyen náuseas severas, vómito, hipotrauma abdominal cerrado, resucitación cardiopulmonar, tos, gritos, barotrauma y convulsiones; en nuestra serie fueron menos frecuentes que en otras publicaciones ( $8 \%$ ) y los múltiples factores predisponentes explicarían esa diferencia $(10,33)$. No obstante, sería importante disminuir al máximo los factores relacionados, ya que el DMW puede profundizarse y producir ruptura transmural, lo que conduce al síndrome de Boeerhäve (34).
La prevalencia encontrada en este estudio para úlcera gástrica fue del 18,18\% $(\mathrm{n}=28)$. En Eslovenia, Skok y colaboradores en el año 2005, en un estudio de cohorte prospectivo en 486 pacientes, encontraron una prevalencia de úlcera gástrica en 84 pacientes, con una prevalencia del $17,3 \%$ (35). Sin embargo, el requerimiento de tratamiento endoscópico por úlcera gástrica en nuestra población fue solo del 5,8\% (9 pacientes). Un estudio francés observacional retrospectivo entre 2007 a 2012 en 88 pacientes, que evaluó el impacto clínico de la EVDA en pacientes críticos con sospecha de sangrado, encontró que solo el 3,5\% de los pacientes requirió manejo endoscópico para úlcera gástrica (7). Estos resultados son importantes, ya que la EVDA es un procedimiento relativamente costoso, que no está exento de riesgos, todo lo cual se incrementaría al utilizarlo en forma generalizada (35).

En nuestro estudio entre pacientes con HVDA que requirieron terapia endoscópica, las causas no varicosas fueron el doble que las varicosas ( $11,03 \%$ vs. $4,55 \%)$, similar a otros estudios (36). Hubo 17 pacientes con úlcera duodenal $(11,04 \%)$, el cual es la mitad de lo informado en la literatura (29). Desconocemos la razón de la discrepancia, pero pudiera estar relacionado con la prevalencia de Helicobacter pylori (H. pylori), la frecuencia de utilización de IBP profiláctico y el tipo de patología crítica de los pacientes, especialmente ventilación mecánica, coagulopatías activas, enfermedad hepática y enfermedad renal. En nuestro estudio, muchos pacientes tenían las patologías predisponentes mencionadas y la utilización de IBP profilácticos fue del $32 \%$ de los pacientes. Los IBP profilácticos han demostrado que disminuyen significativamente los sangrados clínicamente importantes comparados con el placebo $(2,5 \%$ vs. $4,2 \%)$ y son recomendados en esas circunstancias $(2,37)$. 
Muchos médicos aún tienen temor a utilizar los IBP de manera profiláctica por los riesgos teóricos sobre posibles efectos adversos, como neumonía, isquemia miocárdica, C. difficile, entre otros (15). Sin embargo, la evidencia que soporta estas afirmaciones es muy débil y hasta el momento solo se ha establecido asociación y no causalidad (38). En nuestro estudio, la duodenitis erosiva fue superior a lo informado en la literatura ( $21,43 \%$ vs. $6 \%)$, sin embargo, no se identificó sangrado activo con esa patología en nuestros pacientes $(29,34)$.

El presente estudio tiene limitaciones, tales como ser retrospectivo y, debido a las múltiples patologías concomitantes y diagnósticos cambiantes, no fue fácil determinar los factores de riesgo que predisponen a sangrados altos, que ameritarían tratamiento endoscópico y así evitar el 85\% de EVDA innecesarias.

En conclusión, el 15\% de los pacientes necesitó terapia endoscópica en los pacientes con HVDA de cuidados intensivos. Se necesitan trabajos prospectivos, de preferencia multicéntricos, que permitan identificar los factores de riesgo que puedan predecir la necesidad de EVDA terapéutica en pacientes con HVDA. En los pacientes que no tengan esos predictores se recomendaría tratarlos empíricamente con IBP y evitar gastos innecesarios en EVDA diagnósticas. Hasta el momento, en la literatura publicada se han identificado los pacientes con riesgo de HVDA hospitalizados en UCI, pero no los que, teniendo sangrado, podrían necesitar una EVDA terapéutica.

\section{Conflicto de intereses}

Ninguno.

\section{Fuente de financiación}

Los costos de la presente investigación fueron asumidos por los investigadores y la Unidad de Gastroenterología de Clínica Fundadores, una institución de tercer nivel adscrita al posgrado de Gastroenterología de la Universidad Nacional de Colombia (UN).

\section{Agradecimientos}

Agradecemos de manera muy especial a Liliana Oino, Ingeniera Biomédica, coordinadora administrativa de la Unidad de Gastroenterología de la Clínica Fundadores y del convenio Docente-Asistencia UN-Clínica Fundadores por su interés, entusiasmo y colaboración permanente en todas las fases de este estudio, facilitando toda la información sobre procedimientos endoscópicos e identificación de las historias clínicas de los pacientes incluidos. Al doctor Jairo Antonio Pérez Cely, Intensivista del Hospital
Universitario Nacional de Colombia, por la lectura crítica del manuscrito y las sugerencias sobre el mismo.

\section{REFERENCIAS}

1. Marik P, Vasu T, Hirani A, Pachinburavan M. Stress ulcer prophylaxis in the new millennium: a systematic review and meta-analysis. Crit Care Med. 2010;38(11):2222-8. doi: https://doi.org/10.1097/CCM.0b013e3181f17adf.

2. Krag M, Perner A, Wetterslev J, Wise M, Borthwick M, Bendel S, et al. Stress ulcer prophylaxis in the intensive care unit: an international survey of 97 units in 11 countries. Acta Anaesthesiol Scand. 2015;59(5):576-85. doi: https:// doi.org/10.1111/aas.12508.

3. Krag M, Perner A, WetterslevJ,Wise M, BorthwickM, Bendel $S$, et al. Prevalence and outcome of gastrointestinal bleeding and use of acid suppressants in acutely ill adult intensive care patients. Intensive Care Med. 2015;41(5):833-45. doi: https://doi.org/10.1007/s00134-015-3725-1.

4. Cook D, Griffith L, Walter S, Guyatt G, Meade M, Heyland $\mathrm{DK}$, et al. The attributable mortality and length of intensive care unit stay of clinically important gastrointestinal bleeding in critically ill patients. Crit Care. 2001;5(6):368. doi: https://doi.org/10.1186/cc1071.

5. Cook D, Fuller H, Guyatt G, Marshall J, Leasa D, Hall R, et al. Risk factors for gastrointestinal bleeding in critically ill patients. N Engl J Med. 1994;330(6):377-81. doi: https:// doi.org/10.1056/NEJM199402103300601.

6. Cipolletta L, Cipolletta F, Granata A, Ligresti D, Barresi L, Tarantino I, et al. What is the best endoscopic strategy in acute non-variceal gastrointestinal bleeding? Curr Treat Optios Gastro. 2018;16(4):363-75. doi: https://doi. org/10.1007/s11938-018-0192-0.

7. Jean-Baptiste S, Messika J, Hajage D, Gaudry S, Barbieri J, Duboc $\mathrm{H}$, et al. Clinical impact of upper gastrointestinal endoscopy in critically ill patients with suspected bleeding. Ann Intensive Care. 2018;8(1):75. doi: https://doi. org/10.1186/s13613-018-0423-5.

8. Barkun A, Bardou M, Kuipers E, Sung J, Hunt R, Martel $\mathrm{M}$, et al. International consensus recommendations on the management of patients with nonvariceal upper gastrointestinal bleeding. Ann Inter Med. 2010;152(2):101-13. doi: https: / / doi.org/10.7326/0003-4819-152-2-20100119000009.

9. Lau J, Barkun A, Fan DM, Kuipers E, Yang YS, Chan F. Challenges in the management of acute peptic ulcer bleeding. Lancet. 2013;381(9882):2033-43. doi: https://doi. org/10.1016/S0140-6736(13)60596-6.

10. Chak A, Cooper G, Lloyd L, Kolz C, Barnhart B, Wong R. Effectiveness of endoscopy in patients admitted to the intensive care unit with upper GI hemorrhage. Gastrointest Endoscop. 2001;53(1):6-13. doi: https://doi.org/10.1067/ mge.2001.108965.

11. Lee YC, Wang HP, Wu MS, Yang CS, Chang YT, Lin JT. Urgent bedside endoscopy for clinically significant upper gastrointestinal hemorrhage after admission to the intensive 
care unit. Intensive Care Med. 2003;29(10):1723-8. doi: https://doi.org/10.1007/s00134-003-1921-x.

12. Tam W, Bertholini D. Tension pneumoperitoneum, pneumomediastinum, subcutaneous emphysema and cardiorespiratory collapse following gastroscopy. Anaesth Intensive Care. 2007;35(2):307-9.

13. Conrad SA. Acute upper gastrointestinal bleeding in critically ill patients: causes and treatment modalities. Crit Care Med. 2002;30(6):S365-S8. doi: https://doi. org/10.1097/00003246-200206001-00006.

14. Eddleston J, Pearson R, Holland J, Tooth J, Vohra A, Doran $\mathrm{BH}$. Prospective endoscopic study of stress erosions and ulcers in critically ill adult patients treated with either sucralfate or placebo. Critic Care Med. 1994;22(12):1949-54. doi: https://doi.org/10.1097/00003246-199422120-00010.

15. Marker S, Krag M, Møller M. What's new with stress ulcer prophylaxis in the ICU? Intensive Care Med. 2017;43(8):1132-4. doi: https://doi.org/10.1007/s00134017-4733-0.

16. Granholm A, Lange T, Anthon C, Marker S, Krag M, Meyhoff T, et al. Timing of onset of gastrointestinal bleeding in the ICU: protocol for a preplanned observational study. Acta Anaesth Scand. 2018. doi: https://doi.org/10.1111/ aas.13144.

17. Plummer M, Blaser A, Deane A. Stress ulceration: prevalence, pathology and association with adverse outcomes. Crit Care. 2014;18(2):213. doi: https://doi.org/10.1186/ cc13780.

18. Steinberg K. Stress-related mucosal disease in the critically ill patient: risk factors and strategies to prevent stress-related bleeding in the intensive care unit. Crit Care Med. 2002;30(6):S362-S4. doi: https://doi. org/10.1097/00003246-200206001-00005.

19. Siddiqui F, Ahmed M, Abbasi S, Avula A, Siddiqui A, Philipose J, et al. Gastrointestinal bleeding in patients with acute respiratory distress syndrome: a national database analysis. J Clin Med Res. 2019;11(1):42-8. doi: https://doi. org/10.14740/jocmr3660.

20. Alvarado J. Profilaxis de sangrado digestivo en la Unidad de Cuidados Intensivos. Univ Med. 2002;43(1):33-5.

21. Schuster D, Rowley H, Feinstein S, McGue M, Zuckerman G. Prospective evaluation of the risk of upper gastrointestinal bleeding after admission to a medical intensive care unit. Am J med. 1984;76(4):623-30. doi: https://doi. org/10.1016/0002-9343(84)90286-9.

22. Huang HB, Jiang W, Wang CY, Qin HY, Du B. Stress ulcer prophylaxis in intensive care unit patients receiving enteral nutrition: a systematic review and meta-analysis. Crit Care. 2018;22(1):20. doi: https://doi.org/10.1186/s13054-0171937-1.

23. Beejay U, Wolfe M. Acute gastrointestinal bleeding in the intensive care unit: the gastroenterologist's perspective. Gastroenterol Clin North Am. 2000;29(2):309-36. doi: https://doi.org/10.1016/S0889-8553(05)70118-7.

24. Morales C, Sierra S, Hernández A, Arango A, López G. Hemorragia digestiva alta: factores de riesgo para mor- talidad en dos centros urbanos de América Latina. Rev Esp Enferme Dig. 2011;103(1):20-4. doi: https://doi. org/10.4321/S1130-01082011000100004.

25. Lewis J, Shin E, Metz D. Characterization of gastrointestinal bleeding in severely ill hospitalized patients. Crit Care Med. 2000;28(1):46-50. doi: https://doi. org/10.1097/00003246-200001000-00007.

26. Kim J, Kim J, Chun J, Lee C, Im J, Kim J. Early versus late bedside endoscopy for gastrointestinal bleeding in critically ill patients. Korean J Intern med. 2018;33(2):304-12. doi: 10.3904/kjim.2016.182.

27. Hayden S, Albert T, Watkins T, Swenson E. Anemia in critical illness: insights into etiology, consequences, and management. American J Respir Crit Care Med. 2012;185(10):1049-57. doi: https://doi.org/10.1164/ rccm.201110-1915CI.

28. Sesler J. Stress-related mucosal disease in the intensive care unit. AACN Advanced Crit Care. 2007;18(2):119-28. doi: https://doi.org/10.4037/15597768-2007-2004.

29. Silverstein F, Gilbert D, Tedesco F, Buenger N, Persing J. The national ASGE survey on upper gastrointestinal bleeding: II. Clinical prognostic factors. Gastrointest Endoscop. 1981;27(2):80-93. doi: https://doi.org/10.1016/S00165107(81)73156-0.

30. van Leerdam M. Epidemiology of acute upper gastrointestinal bleeding. Best Pract Res Clin Gastroenterol. 2008;22(2):209-24. doi: https://doi.org/10.1016/j. bpg.2007.10.011.

31. Ovenden C, Plummer M, Selvanderan S, Donaldson T, Nguyen N, Weinel L, et al. Occult upper gastrointestinal mucosal abnormalities in critically ill patients. Acta Anaesthesiol Scand. 2017;61(2):216-23. doi: https://doi. org/10.1111/aas.12844.

32. Rich K. Overview of Mallory-Weiss syndrome. J Vasc Nurs. 2018;36(2):91-3. doi: https://doi.org/10.1016/j. jvn.2018.04.001.

33. Guelrud M. Mallory-Weiss syndrome. UptoDate. 2017. Último acceso: 15 de junio de 2018. Disponible en: https:// www.uptodate.com/contents/mallory-weiss-syndrome

34. Cucci M, Capputo F, Fraternali G, Roncallo A, Ventura F. Transition of a Mallory-Weiss syndrome to a Boerhaave syndrome confirmed by anamnestic, necroscopic, and autopsy data. A case report. Medicine (Baltimore). 2018;97(49):e13191. doi: https://doi.org/10.1097/ MD.0000000000013191.

35. Skok P, Sinkovič A. Upper gastrointestinal haemorrhage: predictive factors of in-hospital mortality in patients treated in the medical intensive care unit. J Int Med Res. 2011;39(3):1016-27. doi: https://doi. org/10.1177/147323001103900337.

36. Gralnek I, Dumonceau J, Kuipers E, Lanas A, Sanders D, Kurien M, et al. Diagnosis and management of nonvariceal upper gastrointestinal hemorrhage: European Society of Gastrointestinal Endoscopy (ESGE) Guideline. Endoscopy. 2015;47(10):a1-a46. doi: https://doi. org/10.1055/s-0034-1393172. 
37. Krag M, Marker S, Perner A, Wetterslev J, Wise M, Schefold J, et al. Pantoprazole in patients at risk for gastrointestinal bleeding in the ICU. N Engl J Med. 2018;379(23):2199-208.

38. Rehman A, Iscimen R, Yilmaz M, Khan H, Belsher J, Gomez JF, et al. Prophylactic endotracheal intubation in critically ill patients undergoing endoscopy for upper GI hemorrhage. Gastrointest Endoscop. 2009;69(7):e55-e9. doi: https:// doi.org/10.1016/j.gie.2009.03.002. 